\title{
OCORRÊNCIA DE Amblyomma aureolatum (PALLAS, 1772) (Acari: Ixodidae) EM CACHORRO-DO-MATO (Cerdocyon thous) (LINNAEUS, 1766), NA REGIÃO DO BIOMA PAMPA
}

\author{
PINTO, Diego Moscarelli ${ }^{1}$; \\ AGUIAR, Cíntia Lidiane Guidotti ${ }^{2}$; \\ MARTINS, Natália Soares ${ }^{3}$; \\ BECKER, Marcelo ${ }^{4}$; \\ DAMBORIARENA, Pedro Araújo ${ }^{5}$; \\ TAVARES, Nicolas Conter ${ }^{6}$; \\ ANTUNES, Tatiana de Ávila ${ }^{7}$; \\ SANTOS, Thais Cozza ${ }^{8}$.
}

\footnotetext{
${ }^{1}$ Professor Adjunto do Departamento de Veterinária Preventiva/UFPEL; ${ }^{2}$ Bióloga, Mestre pelo Programa de PósGraduação em Veterinária/UFPEL; ${ }^{3}$ Médica Veterinária, Mestre pelo Programa de Pós-Graduação em Parasitologia/UFPEL; ${ }^{4}$ Médico Veterinário, Mestrando pelo Programa de Pós-Graduação em Saúde Animal/FEPAGRO; ${ }^{5}$ Médico Veterinário; ${ }^{6}$ Médico Veterinário, Mestrando pelo Programa de Pós-Graduação em Zootecnia/UFPEL; 7 Médica Veterinária, Doutora pelo Programa de Pós-Graduação em Veterinária/UFPEL; ${ }^{8}$ Médica Veterinária, Residente em Imaginologia Veterinária pelo Programa de Residência Multiprofissional/UFPEL.
}

\section{RESUMO}

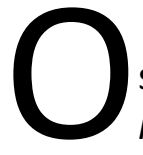

carrapato Amblyomma aureolatum tem extrema importância em saúde pública, sendo considerado, depois do A. cajennense, o principal transmissor de Rickettsia rickettsii, agente causador da Febre Maculosa Brasileira. O presente estudo teve como objetivo relatar o caso de parasitismo por $A$. aureolatum em cachorro-do-mato (Cerdocyon thous) na região do Bioma Pampa, Rio Grande do Sul, Brasil. O animal foi

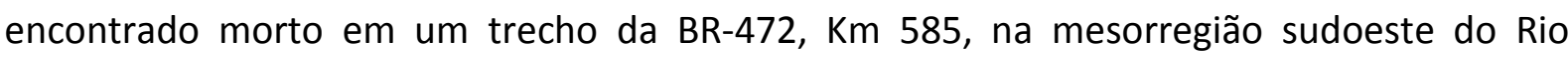
Grande do Sul, e submetido à necropsia. Foram encontrados e identificados, quatro espécimes de carrapatos, todos machos adultos de $A$. aureolatum. A presença de $A$. aureolatum, no exemplar de cachorro-do-mato de vida livre gera algumas questões pertinentes para futuros estudos, tais como a sazonalidade destes parasitos e a possibilidade de infestação e transmissão de doenças para animais domésticos e para o homem, considerando a proximidade principalmente nas propriedades rurais.

Palavras-chave: Carrapato. Animais silvestres. Parasitismo. 


\section{INTRODUÇÃO}

Os carrapatos transmitem maior variedade de agentes patogênicos do que qualquer outro grupo de artrópodes hematófagos, sendo a maioria dos patógenos transmitidos aos vertebrados, principalmente via saliva. No momento em que o carrapato realiza o repasto sanguíneo no hospedeiro, injeta também saliva no local da picada, que possui toxinas ativas à maioria dos vertebrados e também substâncias anestésicas e anticoagulantes. Os carrapatos são, primariamente, ectoparasitos de animais silvestres e a maioria dos vertebrados terrestres está sujeita ao seu ataque (LABRUNA et al., 2005).

Amblyomma apresenta aproximadamente 106 espécies de carrapatos em todo o mundo. Destas, 33 podem ser encontradas no Brasil, parasitando aves, mamíferos, répteis e anfíbios. O carrapato Amblyomma aureolatum habita regiões de floresta Atlântica do sudeste do Brasil, sendo encontrado também no nordeste da Argentina, Guiana Francesa, leste do Paraguai, Suriname e Uruguai. Os principais hospedeiros da fase adulta de $A$. aureolatum são carnívoros silvestres, entre eles, canídeos como o cachorro-do-mato (Cerdocyon thous), a raposa-do-campo (Lycalopex gymnocercus) e a raposinha (Lycalopex vetulus); procionídeos, como o mão-pelada (Procyon cancrivorus) (LABRUNA et al., 2005); e herbívoros como a capivara (Hydrochoerus hydrochaeris) (QUADROS et al., 2015). As formas imaturas deste carrapato são comumente encontradas em aves silvestres e, esporadicamente, em mamíferos de pequeno porte, como roedores e marsupiais (GUIMARÃES et al., 2001; OGRZEWALSKA et al., 2008).

Em áreas rurais ou semiurbanas localizadas próximas a fragmentos de matas com ocorrência de A. aureolatum, sua forma adulta parasita principalmente cães domésticos (GUGLIELMONE et al., 2003). Devido ao frequente acesso de cães no interior de matas, esses hospedeiros tornam-se importantes carreadores de carrapatos adultos para áreas domiciliares, sendo a principal forma de contato desses ectoparasitos com os seres humanos (OGRZEWALSKA et al., 2008). A. aureolatum tem importância, também, na saúde pública, sendo considerado, depois do A. cajennense, o principal transmissor de Rickettsia rickettsii, agente da mais importante zoonose transmitida por carrapatos da América Latina, chamada no Brasil de Febre Maculosa Brasileira (FMB) ou febre do carrapato (LABRUNA, 2009). 
Devido à ação antrópica, vários animais silvestres têm se adaptado ao ambiente semiurbano, entre eles, o C. thous, sendo este aparentemente tolerante a perturbações em seu habitat, possibilitando maior proximidade aos seres humanos. Este canídeo possui ampla distribuição na América do Sul, ocorrendo desde o Uruguai e norte da Argentina até na Bolívia, Venezuela, Colômbia, Guianas, Suriname e Brasil (REIS et al., 2006). C. thous está presente em todos os biomas brasileiros e em uma ampla variedade de habitats, sendo considerado menos preocupante na lista de espécies ameaçadas (BEISIEGEL et al., 2013). Embora possua pouco risco de extinção, é uma das espécies de mamíferos que sofre mais atropelamentos no Rio Grande do Sul (ROSA; MAUHS, 2004).

Diante do exposto, e devido ao aumento de relatos sobre o parasitismo de A. aureolatum no sul do Brasil, torna-se muito importante conhecer a ocorrência desses ectoparasitos e seus hospedeiros silvestres, para com este conhecimento, prevenir a transmissão de algumas doenças de importância médica e veterinária. Neste sentido, o presente trabalho teve como objetivo relatar o parasitismo de A. aureolatum em cachorro-do-mato, na região do Bioma Pampa, Rio Grande do Sul, Brasil.

\section{RELATO DE CASO}

Um canídeo silvestre foi encontrado morto em um trecho de rodovia que cruza o Bioma Pampa, no município de Uruguaiana, Rio Grande do Sul, mais precisamente na BR-472, Km 585 ( $27^{\circ} 34^{\prime} 19.49^{\prime \prime} \mathrm{S}$ e $53^{\circ} 58^{\prime} 21.33^{\prime \prime}$ O; 423 m alt.) na mesorregião sudoeste do Rio Grande do Sul. O canídeo foi recolhido pelo Batalhão Ambiental da Brigada Militar e encaminhado ao Laboratório de Patologia Veterinária da Universidade Federal do Pampa (UNIPAMPA), onde foi identificado, por meio da pelagem e conformação do crânio, como Cerdocyon thous (MAXIMIANO NETO, 2017; REIS et al., 2006). O esqueleto encontra-se na coleção do Laboratório de Anatomia da UNIPAMPA. Durante o exame clínico externo de $C$. thous foram encontrados quatro carrapatos, estes foram enviados ao Laboratório de Parasitologia da UNIPAMPA para análise. Após a identificação em nível de gênero, os espécimes coletados foram encaminhados para o Laboratório de Parasitologia da Universidade Federal de Pelotas (UFPEL), para identificação das espécies dos carrapatos. A identificação dos ixodídeos foi realizada a partir da utilização de chave dicotômica, assim como a verificação de estruturas específicas e estampas de figuras descritas em Barros-Battesti (2006). 


\section{RESULTADOS E DISCUSSÃO}

Foram identificados quatro espécimes, todos machos adultos. Após serem analisados em lupas, e as características morfológicas serem relacionadas às informações presentes na chave e nas figuras, ocorreu a identificação da espécie: Amblyomma aureolatum (BARROSBATTESTI, 2006).

Dentre as características mais importantes para a identificação da espécie, está a presença de espinhos na região ventral. Na coxa I observa-se dois espinhos longos, subiguais e contíguos, sendo que o espinho externo termina em ponta reta (diferentemente do que ocorre com A. ovale que é ligeiramente curvo), já na coxa IV visualiza-se um único espinho (Figura 1).

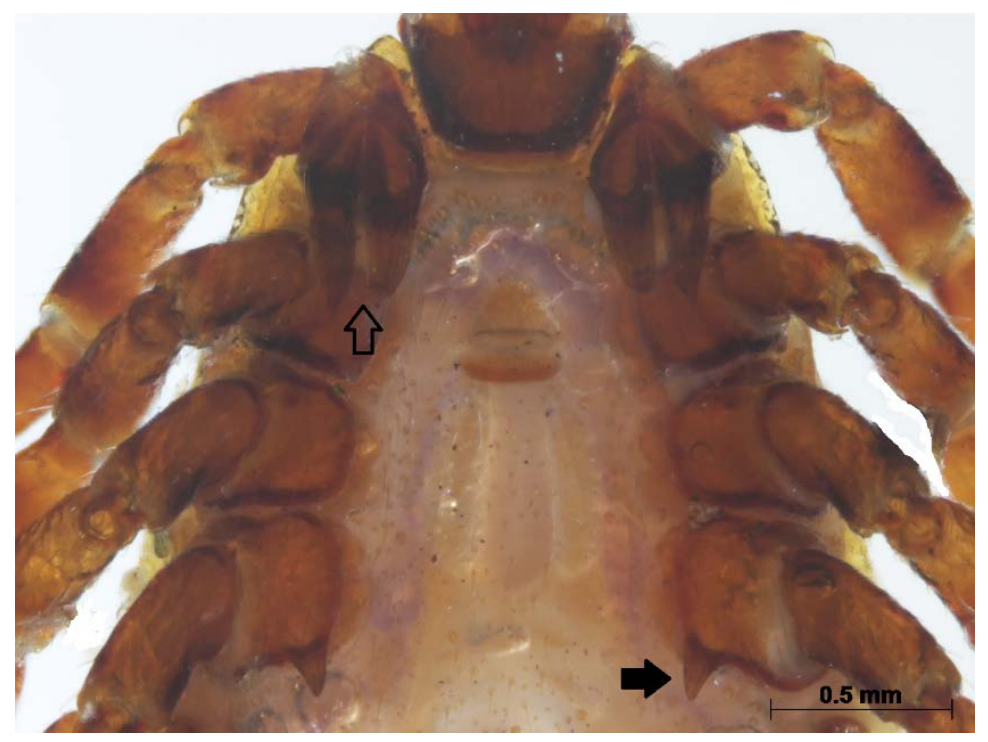

Figura 1 - Macho de Amblyomma aureolatum encontrado em Cerdocyon thous. Posição ventral, em destaque na coxa I dois espinhos longos terminados em ponta reta e na coxa IV (seta preta) espinho único.

Na face dorsal do A. aureolatum observa-se o escudo castanho claro, predominando o colorido amarelo-dourado e com pontuações igualmente distribuídas, além de sulco marginal incompleto alcançando no máximo o segundo festão (Figura 2). 


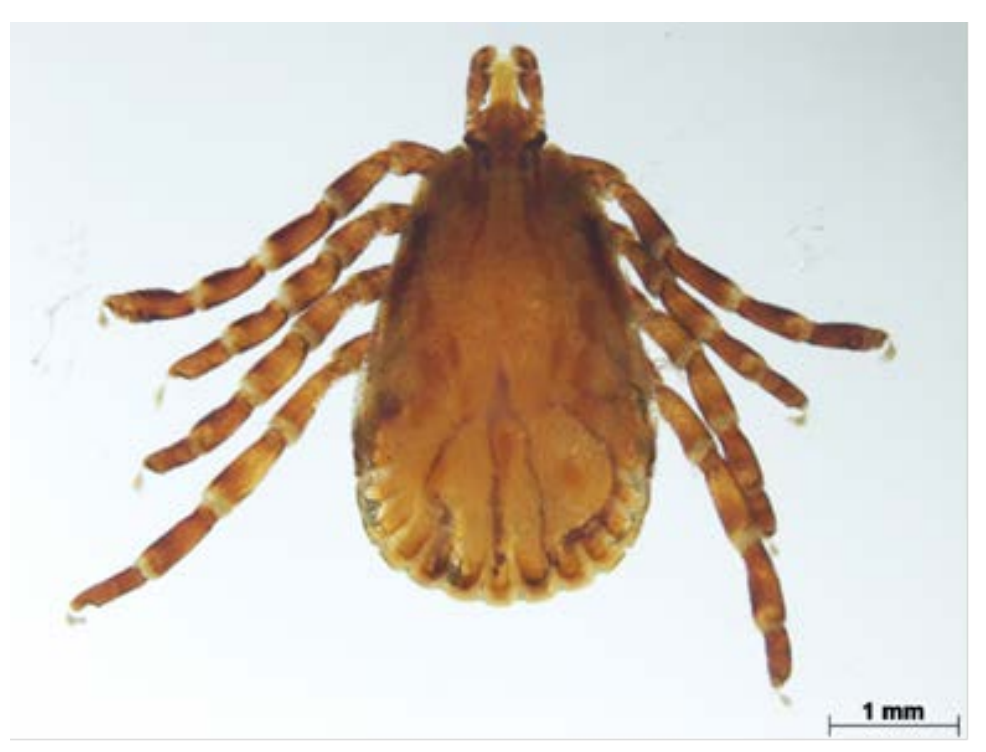

Figura 2 - Macho de Amblyomma aureolatum encontrado em Cerdocyon thous. Escudo dorsal castanho-claro, predominantemente amarelo-dourado e sulco marginal incompleto.

Estudos mostram que os parasitos do gênero Amblyomma são os principais ixodídeos de canídeos silvestres no Brasil (BARROS-BATTESTI, 2006). Freire (1972) relatou que os cachorros-do-mato, assim como os cães domésticos, representam os hospedeiros preferenciais para as espécies de Amblyomma, destacando uma elevada prevalência de $A$. tigrinum.

No estado do Rio Grande do Sul, em levantamento da fauna ixodológica (Freire, 1972), foram identificados nos cães nove espécies de carrapatos: A. tigrinum, A. ovale, $A$. aureolatum, A. cajennense, A. maculatum, A. humerale, A. incisum, Rhipicephalus (Boophilus) microplus e Rhipicephalus sanguineus. Evans et al. (2000) relataram a presença de $A$. aureolatum em $C$. thous no município de Guaíba, localizado na região metropolitana de Porto Alegre/RS. Em Cachoeira do Sul/RS, Martins et al. (2006) relataram a presença de $A$. aureolatum parasitando uma espécie de bugio (Alouatta guariba). Massard et al. (1981), ao estudarem as parasitoses de cães, no período 1976-1980, no Rio Grande do Sul e em alguns outros estados brasileiros, como Rio de Janeiro, Espírito Santo e São Paulo, verificaram que os cães dessas regiões eram parasitados, quando criados em áreas rurais, pelos carrapatos A. ovale, A. aureolatum, A. tigrinum e A. cajennense (exceto no Rio Grande do Sul).

Os carrapatos são conhecidos como potenciais transmissores de agentes infecciosos e parasitários, algumas espécies são comprovadamente transmissoras da FMB e da Doença de 
Lyme (GUIMARÃES et al., 2001). O A. aureolatum, pode transmitir a Rickettsia rickettsi, agente causador da FMB em humanos. Este ixodídeo está entre as espécies identificadas parasitando animais silvestres, como Didelphis albiventris, comumente conhecido como gambá-de-orelha-branca (MULLER et al., 2005). Segundo esses mesmos autores, a proximidade cada vez maior dos animais silvestres aos domicílios possibilita a disseminação de parasitos causadores de zoonoses.

Por ser um carrapato trioxeno, ou seja, que exige três hospedeiros para completar seu ciclo de vida fica evidente que os canídeos silvestres presentes em propriedades rurais, ajudam a disseminar esses parasitos na propriedade, podendo causar perdas econômicas devido à espoliação sanguínea e transmissão de patógenos tanto para os animais domésticos, como para o homem (MONTEIRO, 2010).

\section{CONCLUSÃO}

A presença de Amblyomma aureolatum, neste exemplar de cachorro-do-mato (Cerdocyon thous) de vida livre gera algumas questões pertinentes para futuros estudos, tais como a sazonalidade destes parasitos e a possibilidade de infestação e transmissão de doenças para animais domésticos e para o homem, considerando a proximidade principalmente nas propriedades rurais.

Além disso, a importância desta informação está na falta de literatura e relatos na área de parasitologia de animais silvestres, uma vez que, este é o primeiro relato de cachorro-domato parasitado por A. aureolatum na região estudada.

\section{OCCURRENCE OF Amblyomma aureolatum (PALLAS, 1772) (Acari: Ixodidae) IN CRAB-EATING FOX (Cerdocyon thous) (LINNAEUS, 1766), IN THE PAMPA BIOME REGION}

\section{ABSTRACT}

The Amblyomma aureolatum tick is extremely important in public health because it is considered, after $A$. cajennense, the main transmissor of Rickettsia rickettsii, the causative agent of Brazilian Spotted Fever. This study aimed to report the case of a crab-eating fox (Cerdocyon thous) parasitized by $A$. aureolatum which was found dead in the Bioma Pampa region, Rio Grande do Sul, Brazil. A crab-eating fox from the Uruguaiana city was found dead in the road BR-472, Km 585, in the mesoregion southwest of the Rio Grande 
do Sul state. The animal was subjected to necropsy. During the external examination of the animal four specimens of ticks were identified, all of them were adult males of $A$. aureolatum. The presence of $A$. aureolatum in a free-living crab-eating fox encourages relevant issues for further study, such as seasonality of these parasites and the possibility of transmitting diseases and infestation in domestic animals and humans, considering the proximity especially in the farms.

Keywords: Tick. Wild animal. Parasitism.

OCURRENCIA DE Amblyomma aureolatum (PALLAS, 1772) (Acari: Ixodidae) EN ZORRO CANGREJERO (Cerdocyon thous) (LINNAEUS, 1766), EN LA REGIÓN DEL BIOMA PAMPA

\section{RESUMEN}

a garrapata Amblyomma aureolatum tiene una gran importancia en la salud pública, ya que se considera después del $A$. cajennense, el principal transmisor de Rickettsia rickettsii agente causante de La Fiebre Maculosa Brasileña. El presente estudio tuvo como objetivo relatar el caso de parasitismo por $A$. aureolatum en zorro cangrejero (Cerdocyon thous) en la región del Bioma Pampa, Rio Grande do Sul, Brasil. El animal fue encontrado muerto en la ruta BR-472, Km 585, en la meso región suroeste de Rio Grande do Sul, y sometido a la necropsia. Se encontraron e identificaron, cuatro especímenes de garrapatas, todos machos adultos de $A$. aureolatum. La presencia de A. aureolatum, en el ejemplar de zorro cangrejero de vida libre, genera algunas cuestiones pertinentes para futuros estudios, tales como la estacionalidad de estos parásitos y la posibilidad de infestación y transmisión de enfermedades para animales domésticos y para el hombre, considerando la proximidad principalmente en las propiedades rurales.

Palabras clave: Garrapata. Animal salvaje. Parasitismo.

\section{REFERÊNCIAS}

BARROS-BATTESTI, D. M. Carrapatos de importância médico-veterinária da região neotropical: um guia ilustrado para identificação de espécies. São Paulo: ICTTD-3/Instituto Butantan, 2006. 223p. 
BEISIEGEL, B. M.; LEMOS, F. G.; AZEVEDO, F. C.; et al. Avaliação do risco de extinção do Cachorro-do-mato Cerdocyon thous (Linnaeus, 1766) no Brasil. Biodiversidade Brasileira, v. 3, n. 1, p. 138-145, 2013. Disponível em:

<http://www.icmbio.gov.br/revistaeletronica/index.php/BioBR/article/view/380/287>. Acesso em: 25 jul. 2017.

EVANS, D. E.; MARTINS, J. R.; GUGLIELMONE, A. A. A review of the ticks (Acari, Ixodida) of Brazil, their hosts and geographic distribution - 1. the State of Rio Grande do Sul, Southern Brazil. Memórias do Instituto Oswaldo Cruz, v. 95, n. 4, p. 453-470, 2000. Disponível em: $<$ http://www.scielo.br/scielo.php?script=sci_arttext\&pid=S007402762000000400003\&lng=e n\&nrm=iso>. Acesso em: 27 mar. 2018.

FREIRE, J. J. Revisão das espécies da família Ixodidae. Revue de Médecine Vétérinaire, v. 8, p. 1-16, 1972.

GUglielmone, A. A.; ESTRADA-PEÑA, A.; MANGOLD, A. J.; et al. Amblyomma aureolatum (Pallas, 1772) and Amblyomma ovale (Koch, 1844): hosts, distribution and 16S rDNA sequences. Veterinary Parasitology, v. 113, n. 3-4, p. 273-288, 2003.

GUIMARÃES, J. C.; TUCCI, E. C.; BARROS-BATESTTI, D. M. (Eds). Ectoparasitos de importância veterinária. São Paulo: Plêiade, 2001. 213p.

LABRUNA, M. B.; JORGE, R. S.; SANA, D. A.; et al. Ticks (Acari: Ixodida) on wild carnivores in Brazil. Experimental and Applied Acarology, v. 36, n. 1-2, p. 149-163, 2005.

LABRUNA, M. B. Ecology of Rickettsia in South America. Annals of the New York Academy of Sciences, v. 1166, n. 1, p. 156-166, 2009.

MARTINS, J. R.; SALOMÃO, E. L.; DOYLE, R. L.; et al. First Record of Amblyomma aureolatum (Pallas, 1772) (Acari: Ixodidae) parasitizing Alouatta guariba (Humboldt, 1812) (Primata: Atelidae) in Southern Brazil. Revista Brasileira de Parasitologia Veterinária, v. 15, n. 4, p. 203-205, 2006.

MASSARD, C. A.; MASSARD, C. L.; REZENDE, H. E. B.; et al. Carrapatos de cães em áreas urbanas e rurais de alguns estados brasileiros. In: CONGRESSO BRASILEIRO DE PARASITOLOGIA, 6, 1981, Belo Horizonte. RESUMOS. Belo Horizonte: Sociedade Brasileira de Parasitologia, 1981. P. 201.

MAXIMIANO NETO, A. Morfometria do crânio de cachorro-do-mato Cerdocyon thous. Uberlândia: UFU, 2017. 58p. Tese (Doutorado em Ciências Veterinárias), Faculdade de Medicina Veterinária, Universidade Federal de Uberlândia, 2017.

MONTEIRO, S. G. Parasitologia na Medicina Veterinária. São Paulo: Roca, 2010. 356p. 
MULLER, G.; BRUM, J. G. W.; LANGONE, P. Q.; et al. Didelphis albiventris (Lund, 1841), parasitado por Ixodes loricatus (Neumann, 1899), e Amblyomma aureolatum (Pallas, 1772) (Acari: Ixodidae) no Rio Grande do Sul. Arquivos do Instituto Biológico, v. 72, n. 3, p. 319324, 2005. Disponível em:

<http://www.biologico.sp.gov.br/uploads/docs/arq/V72_3/muller.PDF>

OGRZEWALSKA, M.; PACHECO, R. C.; UEZU, A.; et al. Ticks (Acari: Ixodidae) infesting wild birds in an Atlantic Forest area in the state of São Paulo, Brazil, with isolation of Rickettsia from the tick Amblyomma longirostre. Journal of Medical Entomology, v. 45, n. 4, p. 770$774,2008$.

QUADROS, R. M.; MARQUES, S. M. T.; VERONEZI, W. R. Amblyomma aureolatum (PALLAS, 1772) parasitando capivara (Hidrochaeris hidrochaeris) na região do planalto catarinenserelato de caso. Science and Animal Health, v. 3, n. 2, p. 151-158, 2015.

REIS, N, R.; PERACCHI, A. L.; PEDRO, W. A.; et al. Mamíferos do Brasil. Paraná: Editora Saraiva, 2006. 437p.

ROSA, A. O.; MAUHS, J. Atropelamento de animais silvestres na rodovia RS-040. Caderno de Pesquisa Série Biologia, v. 16, n. 1, p. 35-42, 2004.

Autor para correspondência: Natália Soares Martins. Universidade Federal de Pelotas - Campus Universitário Capão do Leão. CEP 96010900 - caixa postal 354, Pelotas (RS), Brasil. nataliasmartins@outlook.com 